\title{
Tracking Continuous Topological Changes of Complex Moving Regions
}

\author{
Hechen Liu \& Markus Schneider* \\ Department of Computer \& Information Science and Engineering \\ University of Florida \\ Gainesville, FL 32611, USA \\ \{heliu, mschneid\}@cise.ufl.edu
}

\begin{abstract}
A moving region whose location and extend change over time can imply topological changes such as region split and hole formation. To study this phenomenon is useful in many applications, e.g. the topology control of wireless sensor networks and emergency handling. It is challenging to detect the topological changes of a moving region since we lack the ability to capture its continuous change of shapes all the time. Moreover, for a complex moving region containing multiple components, it is hard to determine which component before the change corresponds to which component after the change. In this paper, we propose a model to determine topological changes of a complex moving region through snapshots called observations. We introduce a twophase strategy that the first phase partitions the observations into several evaluation units and uniquely maps a unit before the change to exactly one unit after the change; the second phase interprets the topological change by integrating all basic topological changes from evaluation units.
\end{abstract}

\section{Categories and Subject Descriptors}

H.2.8 [Information Systems]: Spatial Databases and GIS

\section{General Terms}

Design

\section{Keywords}

Moving object, complex region, topological change

\section{INTRODUCTION}

Topology is the mathematical study of the properties that are preserved through deformations such as twisting and

*This work was partially supported by the National Science Foundation (NSF) under the grant number NSF-IIS-0812194 and by the National Aeronautics and Space Administration (NASA) under the grant number NASA-AIST-08-0081.

Permission to make digital or hard copies of all or part of this work for personal or classroom use is granted without fee provided that copies are not made or distributed for profit or commercial advantage and that copies bear this notice and the full citation on the first page. To copy otherwise, to republish, to post on servers or to redistribute to lists, requires prior specific permission and/or a fee.

SAC'11 March 21-25, 2011, TaiChung, Taiwan

Copyright 2011 ACM 978-1-4503-0113-8/11/03 ...\$10.00. stretching. The shape deformation of a spatial object may or may not change its topology. A circle is topologically equivalent to an ellipse, while the fact that a circle is split into two half circles is considered as a topological change. Topological changes of spatial objects are useful in many applications in spatial databases and GIS. For example, the extent of a forest fire can grow in a few days and merge with another spot of fire. The knowledge of this event can help fire fighters prevent the spreading of the fire. Considering another example, two identical mobile networking devices were close to each other at the beginning, and the coverage area of them was one connected region. As they were moving to opposite directions, the coverage area became two disconnected circles in a few minutes. Study such topological change can help researchers improve the performance in wireless sensor networks. In the above examples, the forest fire and the coverage area of the mobile devices are considered as spatial objects whose geometries change over time. Such objects are called moving regions.

In the past, several models have been proposed to represent moving objects. However, although being an important category, the moving region has rarely attracted much attention. There are two major problems regarding the study of topological changes of moving regions. First, we are not able to track the continuous deformation of a moving region due to the deficiency of devices. We detect the forest fire by either obtaining the temperatures from sensors or through pictures captured by satellites. However, these devices can merely collect the needed data every few hours and give us "snapshots". Second, for a complex moving region containing multiple components, we cannot tell precisely which component before a topological change corresponds to which component after the change. Thus, it's difficult to formally determine from two consecutive snapshots, without the human intuition, whether a spot of fire disappears, or it merges with another spot of fire.

The goal of this paper is to propose a model that is able to detect the topological changes of a complex moving region. The detection is based on snapshots captured at instants called observations. We formally define basic topological changes between two consecutive observations such as region split and hole formation. To detect topological changes of a complex moving region, we introduce a two-phase strategy. The first phase partitions the observations before and after the change into evaluation units, and uniquely maps a unit before the change to exactly one unit after the change; the second phase interprets the topological changes of the complex region by integrating all basic topological changes 
of all evaluation units. Topological changes involve more observations can then be evaluated in the same way.

The rest of the paper is organized as follows: Section 2 presents available models for moving regions. Section 3 formally discusses the concept of a moving region and its properties. Section 4 gives our method of detecting topological changes of a complex moving region. Section 5 draws conclusions and discusses the future work.

\section{RELATED WORK}

A complex region is a spatial object which consists of several connected components called faces, each of which may or may not have holes inside it [4]. The topological properties of static region objects have been studied a lot, however, in the moving object context, topological changes of moving regions have rarely attracted much attention.

Several approaches have been proposed to model moving objects in spatial databases and GIS $[2,5,6]$. A moving object is often represented as a function from time to space. For implementation purposes, the movement function is often represented as a polyline in the three-dimensional $(2 \mathrm{D}$ +time) space. Sample points are represented as $(x, y, t)$ tuples, and intermediate locations are predicted through linear interpolation [6]. This representation, however, deals with moving points only, without considering the evolving extent of moving regions over time. Thus it is not suitable for studying topological changes of moving regions.

The approach in [1] discusses the problem of graduate change of topological relationship between two objects. This model analyzes the change of topological relationships between simple regions that is result from their movements. The first approach that gives a discrete representation for complex moving regions is introduced in [7]. It represents a complex region object by a number of faces and solves the problem of constructing the complete moving region from a series of snapshots. However, this model does not discuss the topological change of a moving region. Worboy's group studies topological changes of moving regions in wireless sensor networks, such as region_appear, region_merge, hole_appear, and detects these changes with the help of the sensor devices $[8,3]$. Their research is application oriented which has not formally defined all possible topological changes, and the method does not discuss how to map sub-components properly between two snapshots.

\section{MOVING REGIONS}

In this section, we formally discuss what moving regions are and their properties. They build the foundation of detecting topological changes of a complex moving region.

A moving object is described by a function from time to its corresponding spatial object [2]. Similarly, a moving region object shows a mapping from time to a region object. Thus, before we discuss what is a moving region, we first discuss its corresponding spatial data type, i.e. region. A region object consists of one or several connected components called faces, each of which may or may not have holes inside it [4]. Thus we obtain five possible shapes for a region object. If a region is composed by a single face without holes, we call it a simple region. If a region consists of a single face with only one hole, we name it as a a single region with a hole or a ring. If a region consists of a single face with multiple holes inside it, we name it as a single region with multiple holes. If a region

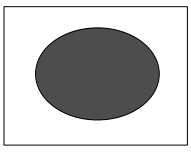

(a)

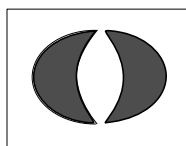

(d)

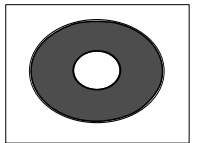

(b)

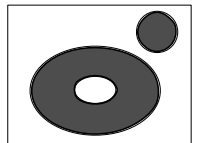

(e)

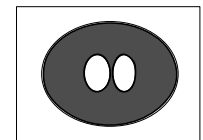

(c)

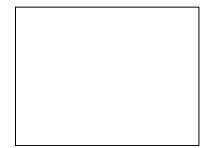

(f)
Figure 1: Simple region without holes (a), single region with one hole (b), single region with multiple holes (c), multi-region without holes (d), complex region (e) and empty region (f).

consists of several faces and for any face there is no hole inside it, this region is called a multi-region without holes. If a region object consists of several faces and some of them have holes, it is called a complex region. In addition, we consider an empty region as a special case of region objects, then we have the sixth possible shapes of a region. The six shapes of regions are illustrated in Figure 1 (a)-(f).

We call the above six shapes of regions as six states of a region object. Since a moving region is a region object evolving over time, the snapshots we take at different time instants can have different states, showing a topological change. For example, an erosion area near the coast becomes two separate areas in ten years. This phenomenon illustrates a topological change that is from a simple region to a multi-region without holes. In contrast, the state of a moving region at different time instants may not change. A hurricane moves from Florida to Louisiana in three days, and the area of the hurricane also changes. However, it remains one simple region without splitting into two parts. This kind of change is called topology-preserving change [3]. In this paper, we mainly focus on the discussion of the internal topological changes of a moving region since they are more important in some applications.

Having discussed the spatial data type region, we now give a formal description of what is a moving region and what properties it has. In our previous work, a moving object is defined as a function from time to a spatial object. In particular, a moving region is defined as $f$ : time $\rightarrow$ region [2]. However, this definition is too general and cannot capture the properties of a movement in reality. For example, under this definition, a simple region can suddenly split into a complex region, but this situation rarely happens in the real world. Only continuous and smooth changes of shapes exist in reality. Thus, a clear definition is needed which is able to describe the continuous changes of a moving region.

We have defined the continuity of a moving region by a slight change of the area between two instants in [2]. Given two regions $R_{1}, R_{2} \in$ region, a concept called the dissimilarity function, which describes the difference between $R_{1}$ and $R_{2}$ in a quantitative way, is defined as $\psi\left(R_{1}, R_{2}\right)=$ $\operatorname{size}\left(R_{1} \backslash R_{2}\right)+\operatorname{size}\left(R_{2} \backslash R_{1}\right)$, where size is the operator to calculate the area of a region object and the $\backslash$ operator denotes the set difference operation between two regions. Take Figure 2(a) for example, a moving region at two different time instants $t$ and $t^{\prime}$ returns two regions $f(t)$ and 


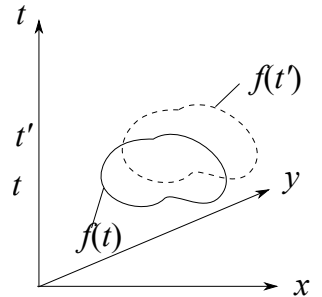

(a)

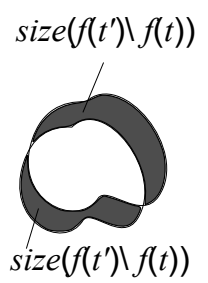

(b)
Figure 2: Snapshots $f(t)$ and $f\left(t^{\prime}\right)$ captured from a moving region at instants $t_{1}$ and $t_{2}$ respectively (a); the dissimilarity area of them in the $2 \mathrm{D}$ plane (b).

$f\left(t^{\prime}\right)$ respectively. $f(t)$ is represented by the shape with the solid line and $f\left(t^{\prime}\right)$ is represented by the one with the dashed line. We observe that $f\left(t^{\prime}\right)$ grows larger and moves to up right of $f(t)$. The projections of them to the 2D plane are shown in Figure 2(b). From Figure 2(b) we observe that most parts of these two regions overlap with each other. The shaded area shows the difference between two regions and thus forms the dissimilarity. Assume that the region changes smoothly, then if the time difference between $t$ and $t^{\prime}$ approaches zero, the dissimilarity area should approach zero accordingly. Based on this idea, we give the definition that describes the continuity of a moving region as follows,

Definition 1 Given a moving region $f:$ time $\rightarrow$ region,

(i) $f$ is right-semicontinuous at $t$, if and only if $\lim _{t^{\prime} \rightarrow t^{+}} \psi\left(\left(f(t), f\left(t^{\prime}\right)\right)=0\right.$

(ii) $f$ is left-semicontinuous at $t$, if and only if $\lim _{t^{\prime} \rightarrow t^{-}} \psi\left(f\left(t^{\prime}\right), f(t)\right)=0$

(iii) $f$ is continuous at $t$, if and only if, it is both rightsemicontinuous and left-semicontinuous at $t$

(iv) $f$ is continuous in $\left[t_{1}, t_{2}\right]$ if, and only if $f$ is right-semicontinuous at $t_{1}$, left-semicontinuous at $t_{2}$ and continuous at any $t \in\left(t_{1}, t_{2}\right)$

Definition $1, f$ is the function of a moving region and $\psi$ denotes the dissimilarity function we have introduced above. Condition (i) describes the situation that if the areal difference between the observation at instant $t$ and the observation at instant $t^{\prime}$ approaches zero, then we call this right-semicontinuous. Since $t^{\prime}$ approaches $t$ from right, we can see that a slight change of time to the future results a slight difference in the area of the moving region, showing the continuity of this movement after instant $t$. Condition (ii) describes the opposite scenario, i.e., if the observation at $t$ changes smoothly with respect to the observation at $t^{\prime}$ right before $t$, then this is left-semicontinuous, showing the continuity of this movement before instant $t$. Condition (iv) discusses the continuity of a moving region during a time interval in which the movement is defined.

Figure 3 illustrates examples of moving regions that are continuous and discontinuous respectively. Figure 3(a) shows a moving region which first shrinks and then grows. The entire changing process is smooth and is considered as a continuous movement. Figure 3(b) shows a region that grows and then forms a hole. Although the topology of the region has been changed, i.e., from a simple region to a single region with holes, the change is continuous and smooth. Figure 3(c) shows an example of discontinuity. At time $t_{2}$, the

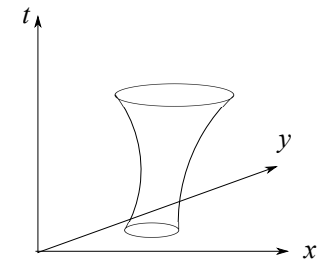

(a)

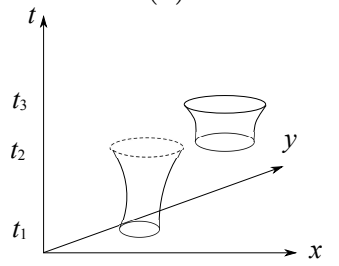

(c)

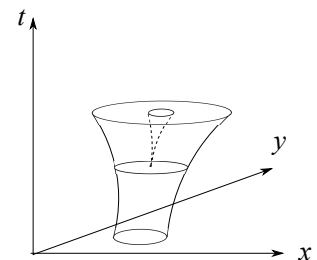

(b)

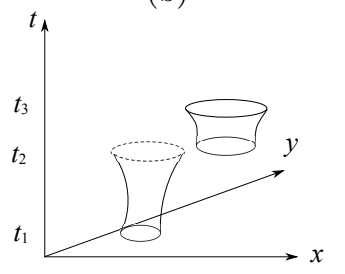

(d)
Figure 3: Moving regions that are continuous during the life domain (a), (b), instantaneous jump (c), and sudden shape change (d)

moving region has an instantaneous jump to a new location, thus the moving region is not left-semicontinuous at $t_{2}$ and thus not continuous in $\left[t_{1}, t_{3}\right]$. In Figure $3(\mathrm{~d})$, the moving region shows a significant topology change at time $t_{2}$, i.e., it changes from a simple region to a complex region, which also violates the continuity.

\section{MODELING TOPOLOGICAL CHANGES OF MOVING REGIONS}

In this section, we present our method for detecting topological changes of a complex moving region through observations. To make the idea convincing that the continuous transition can be detected through snapshots, we make two assumptions. First, the movement of a moving region is considered "continuous", so that there is no instantaneous jump between two consecutive observations, as the case in Figure 3(c). Second, devices such as sensors and satellites update their periodical data with a proper frequency so that there are no "tremendous" topological changes between two observations. Section 4.1 defines basic topological changes. Section 4.2 introduces how to partition observations into evaluation units and map them between two observations. Section 4.3 discusses how to interpret topological changes between consecutive observations.

\subsection{Definitions of Basic Topological Transitions}

A moving region can have different states at different instants, leading to topological changes. However, in reality, topological changes cannot happen between every pair of states. For example, a direct change from a simple region to a complex region is not possible. There must be intermediate states. The state transition diagram representing all direct topological changes is shown in Figure 4, where an arrow shows that direct topological changes are possible between two states. There can be different changes between two same states. For example, from a simple region to a single region with holes, either a hole is formed inside the region, or the region touches itself at two ends. They are named as hole form and region self-touch respectively.

We introduce the concept StateSet, which is the com- 


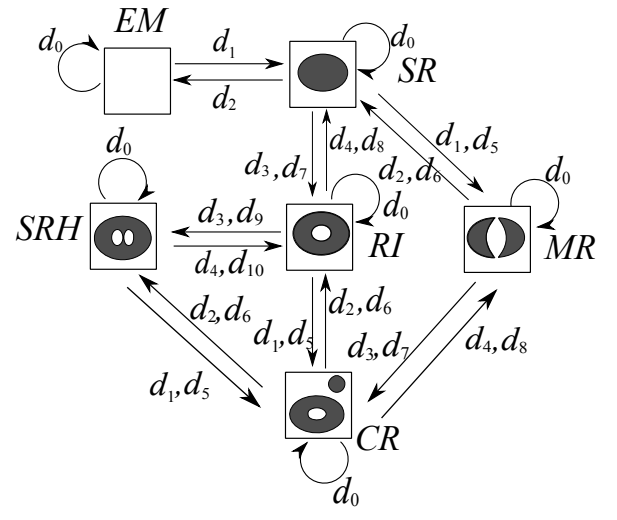

Figure 4: The state transition diagram representing valid topological changes of a moving region

plete set that contains all six states of region objects. Let $E M, S R, R I, S M H, M R, C R$ denote empty, simple region, single region with a hole (ring), single region with multiple holes, multi-region without holes, and complex region respectively, then StateSet $=\{E M, S R, R I, S M H, M R, C R\}$. Now we give the definitions of 11 basic topological changes.

Definition $2 A$ basic topological change of a moving region, denoted by $d_{i}$, is a transition process between two states $S_{1}, S_{2} \in$ stateSet, denoted by $S_{1} \rightarrow S_{2}$, where $t\left(S_{1}\right)<t\left(S_{2}\right)$. The 11 basic topological changes are defined as,

\begin{tabular}{|c|c|c|}
\hline \multirow{5}{*}{$\begin{array}{l}d_{0} \text { (topology preserve) } \\
\left.d_{1} \text { (region appear }\right)\end{array}$} & $S$ & $\longrightarrow S, S \in$ StateSet \\
\hline & $E M$ & $\longrightarrow S R$ \\
\hline & $R I$ & $\longrightarrow C R$ \\
\hline & $S R$ & $\longrightarrow M R$ \\
\hline & $S R H$ & $\longrightarrow C R$ \\
\hline \multirow[t]{4}{*}{$d_{2}$ (region disappear) } & $S R$ & $\longrightarrow E M$ \\
\hline & $C R$ & $\longrightarrow R I$ \\
\hline & $M R$ & $\longrightarrow S R$ \\
\hline & $C R$ & $\longrightarrow S R H$ \\
\hline \multirow[t]{2}{*}{$d_{3}$ (hole form) } & $S R$ & $\longrightarrow R I$ \\
\hline & $M R$ & $\longrightarrow C R$ \\
\hline \multirow[t]{2}{*}{$d_{4}$ (hole fill) } & $R I$ & $\longrightarrow S R$ \\
\hline & $C R$ & $\longrightarrow M R$ \\
\hline \multirow{3}{*}{$d_{5}$ (region split) } & $S R$ & $\longrightarrow M R$ \\
\hline & $R I$ & $\longrightarrow C R$ \\
\hline & $S R H$ & $\longrightarrow C R$ \\
\hline \multirow[t]{3}{*}{$d_{6}$ (region merge) } & $M R$ & $\longrightarrow S R$ \\
\hline & $C R$ & $\longrightarrow S R H$ \\
\hline & $C R$ & $\longrightarrow R I$ \\
\hline \multirow[t]{2}{*}{$d_{7}$ (region self-touch) } & $S R$ & $\longrightarrow R I$ \\
\hline & $M R$ & $\longrightarrow C R$ \\
\hline \multirow[t]{2}{*}{$d_{8}$ (ring half-split) } & $R I$ & $\longrightarrow S R$ \\
\hline & $C R$ & $\longrightarrow M R$ \\
\hline$d_{9}$ (hole split) & $: R I$ & $\longrightarrow S M H$ \\
\hline$d_{10}($ hole merge $)$ & : $S M H$ & $\longrightarrow R I$ \\
\hline
\end{tabular}

In the above definition, "—" indicates a direct topological change, as shown in Figure $4 . d_{0}$ is a special case that the topology is preserved after the change. Such changes include position changing, growing or shrinking. A basic topological change can appear more than once in the diagram, for example, "region appear" $\left(d_{1}\right)$ can happen from empty to a simple region, or from a ring to a complex region, or from a single region with holes to a complex region, etc. Also, between two states, there can be more than one direct development. If a simple region changes to a multiregion without holes, either a "region-split" $\left(d_{3}\right)$ or a "region appear" $\left(d_{7}\right)$ may happen. Now we define the topological development of a moving region during a period of time, by integrating the basic topological changes in time sequence.

Definition 3 Let $\prec$ denote the operator that connected two basic topological changes ordered by time, then the topological development of a complex moving region $C$, is defined as

$$
\operatorname{dev}(C)=d_{1} \prec d_{2} \prec \ldots \prec d_{n}
$$

with the following conditions,

$$
\begin{aligned}
& \text { (i) } n \in \mathbb{N} \\
& \text { (ii) } \forall 1 \leq i \leq n: d_{i} \in\left\{d_{0}, d_{1}, \ldots, d_{10}\right\} \\
& \text { (iii) } \forall 1 \leq i \leq n-1: t\left(d_{i}\right)<t\left(d_{i+1}\right)
\end{aligned}
$$

\subsection{The Partitioning and Mapping Phase}

The basic topological changes in Definition 2 only involve sub-components of a complex moving region. For example, a component before region split is a simple region while after that it is a multi-region without holes. However, this complex moving region may have many other components, and in those other components, more topological changes such as region merge, or hole form may exist at the same time. We call the component which only involves one basic topological change as a evaluation unit. It is the smallest unit to evaluate topological changes of a complex moving region. This subsection introduces the first phase of our method: partitioning and mapping. This phase contains three steps. The first step adjusts the minimum bounding rectangle of two observations to eliminate topology preserving changes such as growing or location changing. The second step partitions each observation to evaluation units. The third step maps an evaluation unit of the first observation to exactly one evaluation unit of the second observation.

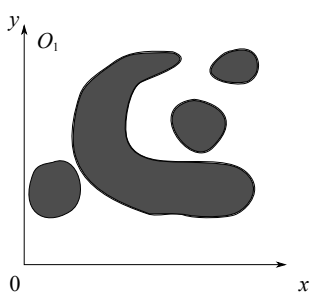

(a)

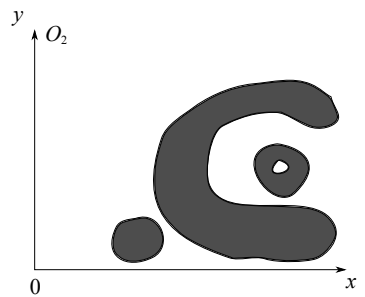

(b)
Figure 5: $O_{1}$ and $O_{2}$ before MBR adjustment

\section{Step1: Adjust Minimum Bounding Rectangles.}

Assume that we are studying the topological change of a forest fire. The observations we take at $t_{1}$ and $t_{2}$ are two complex regions denoted by $O_{1}$ and $O_{2}$ respectively, as shown in Figure 5. Let $\left\langle\left(\min _{i}^{x}, \min _{i}^{y}\right),\left(\max _{i}^{x}, \max _{i}^{y}\right)\right\rangle$ denote the minimum bounding rectangle (MBR) of $O_{i}$, where $i \in\{1,2\}, w_{i}=\max _{i}^{x}-\min _{i}^{x}$ denote the width of the MBR of $O_{i}$, and $h_{i}=\max _{i}^{y}-\min _{i}^{y}$ denote the height of the MBR of $O_{i}$. We adjust the bottom-left point of each rectangle to $(0,0)$ and make the two regions have the same 
MBR. Let $p_{i}=\left(x_{i}, y_{i}\right) \in O_{i}$ denote a point of $O_{i}$ before the adjustment, and $p_{i}^{\prime}=\left(x_{i}^{\prime}, y_{i}^{\prime}\right)$ denote the point after the adjustment. We adjust the MBR of two regions as follows,

$$
\begin{aligned}
& \forall p_{1}=\left(x_{1}, y_{1}\right) \in O_{1}, p_{1}^{\prime}=\left(x_{1}-\min _{1}^{x}, y_{1}-\min _{1}^{y}\right) \\
& \forall p_{2}=\left(x_{2}, y_{2}\right) \in O_{2}, p_{2}^{\prime}=\left(\frac{w_{1}\left(x_{2}-\min _{2}^{x}\right)}{w_{2}}, \frac{h_{1}\left(y_{2}-\min _{2}^{y}\right)}{h_{2}}\right)
\end{aligned}
$$

The MBR of both observations after the adjustment both become $<(0,0),\left(\max _{1}^{x}, \max _{1}^{y}\right)>$, as shown in Figure $7(\mathrm{a}, \mathrm{b})$.

\section{Step2: Partitioning of Complex Regions.}

The partitioning process is shown in Figure 6. This algorithm compares two consecutive observations $O_{1}$ and $O_{2}$, and divides each of them into a list of evaluation units $R_{1}$ and $R_{2}$ respectivelt. The intersection and difference operator in Line 2 and Line 4 denote the geometric set operations of with the same names. We initialize $R_{1}$ and $R_{2}$ to be empty. From Line 2 to Line 3, the algorithm finds the common part of two observations $O$ and adds it to both $R_{1}$ and $R_{2}$. From Line 4 to Line 8 it compares the original observations with $O$, and finds their differences respectively. The difference of an original observation and $O$ is a set of subcomponents, i.e., evaluation units. From Line 8 to Line 16 it adds the sub-components to $R_{1}$ and $R_{2}$ respectively. $R_{1}$ and $R_{2}$ then have the identical geometries with $O_{1}$ and $O_{2}$, however, they are represented as lists of evaluation units instead of two entire complex regions.

\begin{tabular}{|l|c|}
\hline & method partition $(O 1, O 2)$ \\
1 & $R 1 \leftarrow$ null, $R 2 \leftarrow$ null \\
2 & $O \leftarrow$ intersection $(O 1, O 2)$ \\
3 & $R 1 \leftarrow O, R 2 \leftarrow O$ \\
4 & $D 1 \leftarrow$ difference $(O 1, O)$ \\
5 & $m 1 \leftarrow$ size_of $(D 1)$ \\
6 & $D 2 \leftarrow$ difference $(O 2, O)$ \\
7 & $m 2 \leftarrow$ size_of $(D 2)$ \\
8 & for $i=1$ to $m 1$ \\
9 & $R 1 \leftarrow R 1 \cup D 1[i]$ \\
10 & $i \leftarrow i+1$ \\
11 & endfor \\
12 & for $j=1$ to $m 2$ \\
13 & $R 2 \leftarrow R 2 \cup D 2[j]$ \\
14 & $j \leftarrow j+1$ \\
15 & endfor \\
16 & end \\
\hline \multicolumn{2}{|c}{} \\
\hline
\end{tabular}

Figure 6: The partition algorithm that divide observations into evaluation units.

\section{Step3: Mapping Evaluation Units.}

On the basis of the above partitioning result, we perform the following mapping strategies. For each face $c_{1, i} \subset R_{1}$,

- if $c_{1, i}$ is already mapped, proceed to the next element $c_{1, j}$ in $R_{1}$, where $j>i$ and $j$ is the smallest number that satisfy this condition.

- If $c_{1, i}$ is a single face of $R_{1}$, that is, $c_{1, i}$ is not adjacent to any other components, and $c_{2, i}$ is also a single face of $R_{2}$, then we map $c_{1, i}$ to $c_{2, i}$, denoted by $c_{1, i} \rightarrow c_{2, i}$.

- If $c_{1, i}$ is adjacent with other components in $R_{1}$ and $c_{2, i}$ is a single face in $R_{2}$, then we add all components that can be reached from $c_{1, i}$ in $R_{1}$ to the left side of the

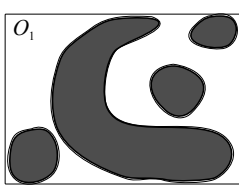

(a)

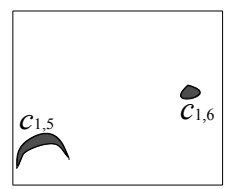

(d)

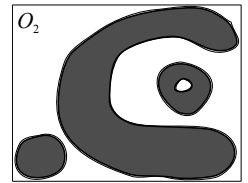

(b)

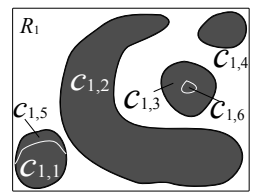

(e)

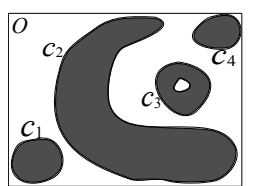

(c)

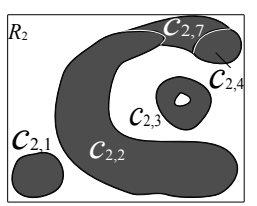

(f)
Figure 7: Adjust MBR (a, b); intersection of two snapshots $O(\mathbf{c})$; difference between $O_{1}$ and $O$ (d); results of the partition on two snapshots $(e, f)$.

arrow; we add $c_{2, i}$ to the right side. If the left side has a component which is also contained in $R_{2}$ but has not been mapped, append it to the right side. For example, assume that in $R_{1}$ we have $c_{1,1}$ that is adjacent to $c_{1,3}$, and $c_{1,3}$ is adjacent to $c_{1,2}$; in $R_{2}$, we have $c_{2,1}$ and $c_{2,3}$ which are disjoint with each other, and we do not have $c_{2,2}$ in $R_{2}$, then we have the following mapping: $c_{1,1} \oplus c_{1,3} \oplus c_{1,2} \rightarrow c_{2,1} \oplus c_{2,3}$, where $\oplus$ denotes the geometric set operation union.

- If $c_{1, i}$ is a single face in $R_{1}$ and $c_{2, i}$ is connected to other components in $R_{2}$, then we add all components that can be reached from $c_{2, i}$ in $R_{2}$ to the right side of the arrow; we add $c_{1, i}$ to the left side. If the right side has a component which is also contained in $R_{1}$ but has not been mapped, we append it to the left side.

- If there are remaining non-mapped single faces $c_{1, i}$ in $R_{1}$ or $c_{2, j}$ in $R_{2}$, then they are mapped to empty on the other side.

To illustrate this partitioning and mapping phase, we take the diagram in Figure 5 for example. It shows observations of a complex moving region at two time instants $O_{1}$ and $\mathrm{O}_{2}$. From the diagrams we notice that this complex region moves to the right and down direction during the interval in between. In addition, we have a few findings of the changes between these two diagrams: 1 . the left bottom component in $O_{1}$ shrinks compared to $O_{2} ; 2 . O_{1}$ has four faces but $O_{2}$ has only three faces, indicating that either a region merge or a region disappear happens; 3 . the component in the middle of $O_{1}$ forms a hole, compared with $O_{2}$. However, they are only intuitive views. Now we explain what happened in a formal way using our method. Our first step is to adjust the minimum bounding rectangles of both observations. This eliminates the fact of a location change or other topology preserving changes and thus enables us to focus on the topological changes. The results of the adjustment are shown in Figure $7(\mathrm{a}, \mathrm{b})$ where two observations have the same MBRs. Then we perform the second step. We apply the intersection operation on $O_{1}$ and $O_{2}$ which returns the overlap part of them, denoted by $O$, as shown in Figure 7(c). After we find the overlap part, we need to find the difference between the two observations. Thus we apply set difference operation between $O_{1}$ and $O$, as well as $O_{2}$ and $O$. The difference between $O_{1}$ and $O$ is shown in Figure 7(d). The left bottom 


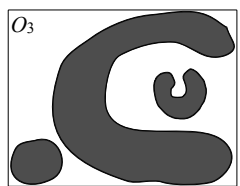

(a)

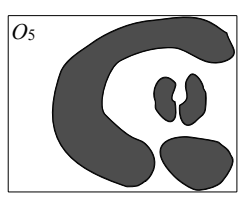

(c)

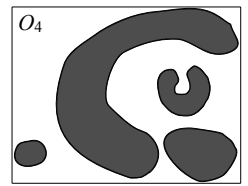

(b)

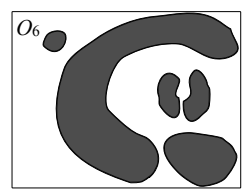

(d)
Figure 8: Later observations of the complex moving region: $\mathrm{O}_{3}(\mathbf{a}), \mathrm{O}_{4}(\mathbf{b}), \mathrm{O}_{5}(\mathbf{c})$ and $\mathrm{O}_{6}(\mathbf{d})$

part $c_{1,5}$ is adjacent to $c_{1,1}$ in $R_{1}$, and $c_{1,1}$ corresponds to $c_{2,1}$ in $R_{2}$. The component $c_{1,6}$ in the middle right of the diagram is the part that disappears in $\mathrm{O}_{2}$, which forms a hole inside $c_{3}$. Then we obtain Figure $7(\mathrm{e})$ which is a new partition of $O_{1}$, and we name it as $R_{1}$. Similarly, we get the new partition of $\mathrm{O}_{2}$, which is $R_{2}$, shown in Figure $7(\mathrm{f})$. We observe that Figure $7(\mathrm{e})$ is identical to Figure $7(\mathrm{a})$, and Figure 7(f) is identical to Figure 7(b) in geometry.

According to the mapping strategy, we obtain the following mappings,

$$
\begin{aligned}
& c_{1,1} \oplus c_{1,5} \rightarrow c_{2,1} \\
& c_{1,2} \oplus c_{1,4} \rightarrow c_{2,2} \oplus c_{2,7} \oplus c_{2,4} \\
& c_{1,3} \oplus c_{6}^{1} \rightarrow c_{2,3}
\end{aligned}
$$

\subsection{The Direct Development Interpretation Phase}

After performing three steps in the partitioning and mapping phase, we obtain the knowledge of which evaluation unit in the first observation is mapped to which evaluation unit in the second observation. Now we are able to explain the transition between two observations by integrating all transitions between all pairs of evaluation units.

In the above example, we obtain the mapping between three pairs of evaluation units. For each mapping, we find a unique one of the 11 basic topological developments corresponding to it, and we have the following translation,

$$
\begin{aligned}
& c_{1,1} \oplus c_{1,5} \rightarrow c_{2,1} \quad: \quad d_{0} \\
& c_{1,2} \oplus c_{1,4} \rightarrow c_{2,2} \oplus c_{2,7} \oplus c_{2,4} \quad: \quad d_{6} \\
& c_{3}^{1} \oplus c_{6}^{1} \rightarrow c_{3}^{2} \quad: \quad d_{3}
\end{aligned}
$$

Since $d_{0}$ is the topology preserving change, we do not need to report this change. Since $d_{6}$ and $d_{3}$ both happen in the interval between $O_{1}$ and $O_{2}$, we do not take care of their ordering. Thus the topological change of the moving region according to the first two snapshots is described as $d_{6} \prec d_{3}$ or $d_{3} \prec d_{6}$, which can be explained as region merge, followed by hole form, or vice versa.

Assume that we have observations of the complex moving region at later instants which are captured in sequence, $\mathrm{O}_{3}, \mathrm{O}_{4}, \mathrm{O}_{5}$ and $\mathrm{O}_{6}$ respectively, as shown in Figure 8. We apply the same strategy to each consecutive pair of observations. Then for each observation pair, we obtain a sequence of topological developments. From $\mathrm{O}_{2}$ to $\mathrm{O}_{3}$ we detect $d_{8}$; from $O_{3}$ to $O_{4}$ we detect $d_{5}$; from $O_{4}$ to $O_{5}$ we detect $d_{2} \prec d_{5}$; from $O_{5}$ to $O_{6}$ we detect $d_{1}$.
In the end we compose all the topological developments between different observations together by the $\prec$ operator. Therefore, from $O_{1}$ to $O_{6}$, we obtain the sequence of topological development,

dev $=d_{6} \prec d_{3} \prec d_{8} \prec d_{5} \prec d_{2} \prec d_{5} \prec d_{1}$

The topological developments are thus explained as region merge, hole form, region half_split, region split, region disappear, region split, region appear.

\section{CONCLUSIONS AND FUTURE WORK}

In this paper, we address an important problem of identifying topological changes of a complex moving region, which can be widely used in many applications, for example, controlling the topology of wireless sensor networks and handling emergency events such as forest fires. We formally discuss the continuity of moving regions. Since it is difficult to track and store the continuous movements, we detect topological changes of complex moving regions through snapshots at different instants called observations. The major problem of finding topological changes from snapshots is that we do not know which component of the region in the snapshots before the change maps to which component after the change. In our method, we give a two-phase strategy which first successfully performs the mapping process and then interprets the topological changes between two consecutive observations and composes all topological development between different observations. In the future we will extend our concept to the topological relationships between different complex moving regions.

\section{REFERENCES}

[1] M. J. Egenhofer and K. K. Al-Taha. Reasoning about gradual changes of topological relationships. In International Conference GIS, pages 196-219, 1992.

[2] R. H. Güting, M. H. Böhlen, M. Erwig, C. S. J. N. A. Lorentzos, M. Schneider, and M. Vazirgiannis. A Foundation for Representing and Querying Moving Objects. ACM Trans. on Database Systems (TODS), 25(1):1-42, 2000.

[3] J. Jiang and M. Worboys. Detecting basic topological changes in sensor networks by local aggregation. In 16th ACM SIGSPATIAL international conference on Advances in geographic information systems, pages 1-10, 2008.

[4] M. Schneider and T. Behr. Topological relationships between complex spatial objects. ACM Trans. on Database Systems (TODS, 31:39-81, 2006.

[5] A. P. Sistla, O. Wolfson, S. Chamberlain, and S. Dao. Modeling and querying moving objects. In 13th International Conference on Data Engineering(ICDE), pages 422-432, 1997.

[6] J. Su, H. Xu, and O. H. Ibarra. Moving Objects: Logical Relationships and Queries. In 7th Int. Symp. on Spatial Temporal Databases (SSTD), pages 3-19, 2001.

[7] E. Tøssebro and R. H. Güting. Creating representations for continuously moving regions from observations. In 7th International Symposium on Advances in Spatial and Temporal Databases, pages 321-344, 2001.

[8] M. Worboys and M. Duckham. Monitoring qualitative spatiotemporal change for geosensor networks. International Journal of Geographical Information Science, 20:1087-1108, 2006. 